\title{
C504 Tomographic Reconstruction of Density Fields Using Modified Algebraic Reconstruction Technique for Laser Speckle Photography Projections
}

\author{
H. S. Ko and Koji Okamoto \\ Nuclear Engineering Research Laboratory \\ University of Tokyo \\ Tokai-mura, Ibaraki, 31911 \\ JAPAN
}

\author{
Kenneth D. Kihm \\ Department of Mechanical Engineering \\ Texas A\&M University \\ College Station, Texas 77843-3123 \\ U.S.A.
}

\begin{abstract}
The reconstructed images from a tomographic reconstruction technique such as an algebraic reconstruction technique (ART) are examined for laser speckle photography. Because of non-algebraic characteristics of line-ofsight projections of speckle photography, the ART is modified to solve this problem. The modified ART converts non-algebraic speckle photographic projection data into algebraic interferometric projections so that the linear ART can proceed. The modified ART of the speckle photography shows good reconstruction results for both of computer synthesized phantom fields and asymmetric helium jets in experiments although it requires an additional step of integration.
\end{abstract}

Keywords: Tomographic Reconstruction, Algebraic Reconstruction Technique, Speckle Photography

\section{Introduction}

Speckle photography (Françon 1979) is a nonintrusive optical technique to detect light ray refraction through an optically thin phase object of varying density field. The technique provides highly accurate measurements with small spatial data resolutions and its wide range of applications to various thermal and fluid engineering problems (Kihm 1997).

Speckle photography is a line-of-sight technique and the ray integral of the information on the refractive index gradient, normal to the ray direction, is projected as beam deflection angle into a point on the projection plane. Using the Gladstone-Dale relation, $n=1+K \rho$, the projected beam deflection angle $\alpha$ is given as a ray integral of the field density gradient:

$$
\psi_{S . P .}(s, \theta) \cong \alpha=K \int \frac{\partial \rho(x, y)}{\partial s} d t
$$

where $s$ is perpendicular to, and $t$ is parallel to the incident ray. The line-of-sight ambiguity in $\alpha$, which occurs from the ray integral along $t$, must be eliminated so that the true density field, $\rho(x, y)$, can be reconstructed. To achieve this, Eq. (1) must be inverted and this mathematical or iterative inversion procedure is called tomography.

Since Eq. (1) correlating the projection and the density field is non-algebraic, the conventional algebraic reconstruction technique (ART) cannot invert Eq. (1). The present work presents a modification of ART where non-algebraic speckle photography data are converted to algebraic interferometric data and the iterative ART reconstruction is carried out for the converted interferometric data. The modified ART has been examined computationally by using a computer-synthesized phantom and experimentally using an asymmetric helium jet.

The ART (Gorden 1974) undertakes the optimization task for the linear case where each basis function is defined by a single parameter (usually its unknown height with a fixed spread) and the location of each basis function is given, i.e.,

$$
\hat{f}(x, y)=\sum_{i=1}^{N} A_{i} b\left(x-x_{i}, y-y_{i}\right)
$$

where $b$ represents a general form of basis function and $A$ is the height coefficient of the $i$-th basis function centered at a specified location of $\left(x_{i}, y_{i}\right)$.

The basic ART uses feedback information on the deviation of the virtual projection from the measured projection and iteratively optimizes the object coefficient vector $A_{i}$ by a simple algebraic updating:

$$
A_{i}^{q+1}=A_{i}^{q}+W_{i}^{q} \frac{\sum_{p}\left(\psi_{p}-\hat{\psi}_{p}\right)}{N P}
$$

where $W_{i}$ is the weighting factor, $q$ denotes the $q$-th iteration, and $N$ is the number of basis functions. An optimized set of unknown $A_{i}$ 's must be found to minimize the deviations between the virtual projection $\hat{\psi}$ of an intermediate object function $\hat{f}$ and the measured projection $\psi$ of the actual field $f$.

2. Line-of-sight projections: laser speckle photography and interferometry

The refractive index variation of a phase object deflects the incident laser beam. The beam deflection angles or its interfered pattern with undeflected beams are projected in a line-of-sight manner on the recording plane. Laser speckle photography records the projected beam deflection angles on a double-exposed photographic film. Wave-front interferometry, such as Mach-Zehnder interferometry, records the interfered fringe patterns.

The beam deflection angle of speckle photography is given as a ray integral of the field density gradient normal to the incident ray, as expressed in Eq. (1). When coherent light is incident upon the random diffraction grating, usually made of ground glass, the light rays randomly diffract in all directions in a constructive or destructive way. When viewed from a distance, the constructive or destructive interferences will be seen as very fine patterns of bright and dark spots, or laser speckles. The change in the index of refraction of the test field will bend the ray slightly from its original path. This causes the incident ray to strike the 
ground glass at a slightly different angle and the speckle dislocates by an amount $\delta$, from its original location.

Wave front interferometry creates the path length difference between the reference beam and the test beam that goes through a phase object. The resulting fringes are then shifted with respect to the undisturbed fringes (fringes formed by the two beams without a tested phase object) and the fringe shift number is expressed as:

$$
\psi_{I . F .}=\frac{1}{\lambda} \int\left(n-n_{r e f}\right) d t=\frac{K}{\lambda} \int_{-\infty}^{\infty}\left(\rho-\rho_{\text {ref }}\right) d t
$$

where $\lambda$ denotes the laser wave length. Note that the fringe shift number is determined directly by the ray integration of the density field, whereas the beam deflection angle of laser speckle photography is given by the ray integration of the density gradient, shown in Eq. (1).

Combining Eqs. (1) and (4) gives

$$
\frac{\partial \psi_{I . F}}{\partial s}=\frac{K}{\lambda} \frac{\partial}{\partial s} \int\left(\rho-\rho_{r e f}\right) d t=\frac{K}{\lambda} \int \frac{\partial \rho}{\partial s} d t=\frac{1}{\lambda} \psi_{S . P .}
$$

Integrating Eq. (5) along $s$ on the projection plane gives

$$
\psi_{\text {I.F. }}=\frac{1}{\lambda} \int \psi_{S . P .} d s
$$

Equation (6) states that an integral of the ray deflection angle, $\psi_{S . P .}$, along $s$ is equivalent to the interferometric fringe shift number $\psi_{I . F}$. Once this conversion to interferometric data is made, the $A \bar{R} T$ operator, Eq. (3), can proceed in updating by using the straightforward feedback information on the density itself.

\section{Reconstruction of test phantoms}

The two reconstruction algorithms, AR' $\mathrm{T}$ and existing Fourier Convolution (FC) method, were used for reconstruction of the phantom field (CosGauss function) (Fig. 1-a) under the speckle projections. The speckle photographic projection of the phantom was simulated by analytically differentiating the functional representation in the normal direction to the ray. For the CosGauss phantom each projection consists of 63 rays $(M=63)$; the object field is described by $25 \times 25$ discrete pixels for the FC reconstruction, and by $25 \times 25$ cubic $\mathrm{B}$-spline basis functions for the ART $(N=625)$. When the ART iteration exceeds the optimum iteration steps, artifacts may violate the convergence resulting in gradually increasing errors (Decker 1993). Thus, ART calculations are ceased when the minimum is reached for the discrepancy between the reconstructed field and the phantom field.

The reconstruction calculations were performed with five equally angled projections $(P=5)$. After the discrete speckle data are curve-fitted by a high-order polynomial function, the numerical integration is carried out with very fine mesh resolution. A trapezoidal rule was used for the numerical integration and no significant numerical integration error is shown to contribute to the overall reconstruction error.

The FC reconstruction of the double-peak phantom of the CosGauss function (Fig. 1-b) shows noisy and erroneous data. In addition to the noise arising near the field boundary, four or more misrepresenting peaks are present. The modified ART reconstruction of the CosGauss phantom (Fig. 1-c) shows significant improvement over the FC reconstruction result of Fig. 1-b. The two peaks are clearly pronounced and the peak locations are accurately reconstructed. The peak heights, however, are 15 to $20 \%$ underpredicted compared with the phantom heights. It is believed that this discrepancy can be attributed primarily to the numerical diffusion due to the discrete representation of the phantom field using the basis function, and numerical integration of the projected data, carried out to convert to interferometric data (Eq. (6)). Nevertheless, the modified ART method shows a noticeably improved reconstruction performance in comparison with the FC method.

\section{Experimental setup}

An asymmetric helium jet from a two-hole orifice (Fig. 2) was tested for the modified ART reconstruction from the speckle photographic projections. The orifice was installed at the end of $12.7 \mathrm{~mm}$ ID copper tube. For detailed positioning, the entire jet unit sits on a rotational, vertically adjustable stand. Line-of-sight speckle photographic images were taken at 6 equally-angled projections with 85 projection data points for the two-hole jet. A Pitot tube measured flow velocity profiles at the tube exit, which converted to the spatially averaged velocity.

The present speckle photography system uses a $35 \mathrm{~mW}$ He-Ne laser $(\lambda=632.8 \mathrm{~nm})$ as the light source. A $4 \times 5$ format camera $(\mathrm{f}=5.6$ at $1 / 250 \mathrm{sec})$ records superimposed speckle images, with and without a test field. The photographic negative of speckle images is mounted to a precise $x-y$ stage and is interrogated by a $10 \mathrm{~mW}$ He-Ne laser with a beam diameter of $0.8 \mathrm{~mm}$. At each illuminated point on the specklegram, Young's fringes are generated and the fringe images are recorded by a CCD camera and digitized into a PC to measure the fringe spacing, $s_{F}$. The beam deflection angle is given as

$$
\alpha=\frac{m}{m^{\prime}} \frac{\lambda d}{c s_{F}}
$$

where $c$ is the defocusing distance (Fig. 3), $d$ is the distance between the specklegram and the imaging screen for Young's fringes, and $m$ and $m$ ' denote the magnification of the parabolic mirror and the camera, respectively. The beam deflection angle, Eq. (7), constitutes the line-of-sight speckle photographic projections of Eq. (1).

An oxygen analyzer directly measures the density of air/helium mixture by detecting the oxygen percentage of sampled gas (Fumizawa and Okamoto 1993). The principle of the analyzer is based on a Zirconia sensor that measures the quantity of oxygen ions at an elevated temperature. The normalized helium concentration, obtained from the oxygen analysis, is used for the results shown in the next section.

\section{Experimental Results}

$25 \times 25$ cubic B-spline basis functions were used to constitute the cross-sectional object field of the two-hole jet. The regression technique fitted the discrete projection data (85 data points per projection) to the 9-th order polynomials and the polynomials were analytically integrated to the interferometric data for the successive ART reconstruction.

In this case, since the reference field is unknown and yet to be determined, the projection error is the only available measure of the reconstruction accuracy. Since the ART can increase artifacts and numerical diffusion when the number of iterations exceeds a certain optimum (Decker 1993), the present ART calculations were carried out until the projection errors reach nearly asymptotic values, i.e., 100 iterations for the double-peak jets. Unlike the phantom testing, where the error of the reconstructed field is available because of the known reference field and the ART iteration can stop at the minimum reconstruction error, the real application of ART is recommended to operate in a way to halt the iteration once the projection error reaches the minimum. 
Figure 4-a shows the ART reconstruction result of normalized helium concentration and Fig. 4-b shows the FC reconstruction result. The reconstruction for all the present cases was carried out for the cross-section located $9.0 \mathrm{~mm}$ above the orifice. The helium jet was maintained laminar at $\operatorname{Re}=1270$ for the two-hole orifice, based on the tube inner diameter $D=12.7 \mathrm{~mm}$.

The results in Fig. 4 show the superior reconstruction capability of the modified ART over FC. The ART reconstruction result shows more accurate density field contours and lower noise than the FC result. The ART result contains virtually no directional dependence and no ambiguities outside the jet regions. The use of cubic Bspline basis functions helps to reconstruct smooth and more comprehensive fields. The FC result, however, shows noisy and immature reconstruction compared with the ART result. The square-pixel construction of the objective field in FC produces the discretized result showing bumps in the field. The FC result also shows a directional dependence with enlarged noise along the projection directions. Non-zero values outside the jet are believed to be the mathematical artifacts due to the only 6 projection data sets that are insufficient for the one-time analytical inversion of FC calculation.

Figure 5 shows the reconstructed results along the symmetric lines, as oriented vertically in Fig. 3, in comparison with the measured helium concentration using the oxygen sensor probe. The solid circles represent the average values of fluctuating readings from the oxygen sensor unit and the fluctuating ranges are marked with the uncertainty bars for the peak reading cases. The modified ART result (solid curve) shows more accurate identification of the peaks and agrees more quantitatively with the oxygen probe results. The $\mathrm{FC}$ result (dashed curve) does not accurately predict the peak locations and slopes.

\section{Concluding remarks}

The line-of-sight projection of the laser speckle photography constitutes the beam deflection data processing diagnostic that measures density and/or temperature fields with superior measurement resolutions. The modified algebraic reconstruction technique (ART) was developed for the purpose of using tomographic reconstruction of speckle photographic images. The modified ART converts nonalgebraic speckle projection data into algebraic interferometric projections so that the linear ART iteration process can proceed. The accuracy and efficacy of the new ART scheme were examined computationally by using the computer synthesized phantom field and experimentally by the use of the asymmetric helium concentration field developed from the orifice nozzle. In comparison with the Fourier convolution (FC) reconstruction, the ART shows significant improvement in reconstruction accuracy and smoothness. Also, the ART results show good agreement with the direct measurement of helium concentration distributions using an oxygen analyzing sensor.

\section{References}

1) Decker, T.J., Tomographic methods in flow diagnostics. NASA Report No. 106330, (1993)

2) Françon, M., Laser speckle and applications in optics. Academic Press, New York, (1979)

3) Fumizawa, M. and Okamoto, K., Mole fraction distribution in a slow jet forming a stably stratified field. Kerntechnik, Vol.58(1993), PP.32-36

4) Gordon, R., Tutorial on algebraic reconstruction technique, IEEE Trans., NS- 21(1974), PP.78-92
5) Kihm, K.D., Laser speckle photography technique applied for heat and mass transfer problems. Advances in Heat Transfer, Vol.30(1997), PP.255-311

6) Verhoeven, D., Limited-data computed tomography algorithms for the physical sciences. Appl. Opt., Vol.32(1993), PP.37363754



(a)

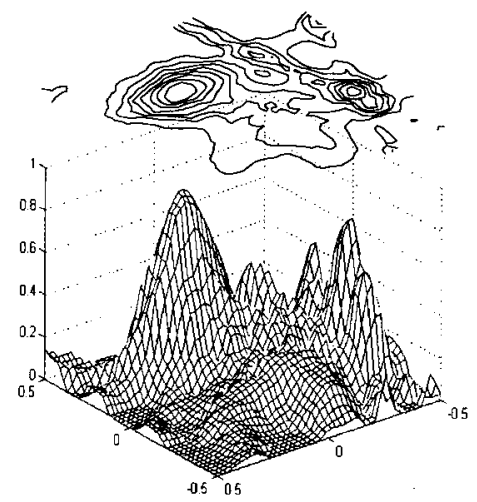

(b)

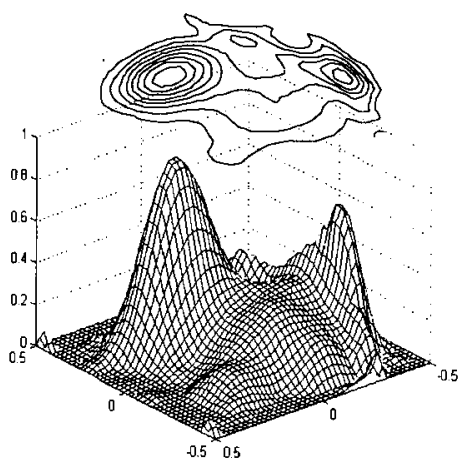

(c)

Figure 1 Reference phantom field and its reconstructions for speckle photography under five projections: (a) CosGauss phantom; (b) FC reconstruction; and (c) ART reconstruction 




Figure 2 Schematic of two-hole nozzle

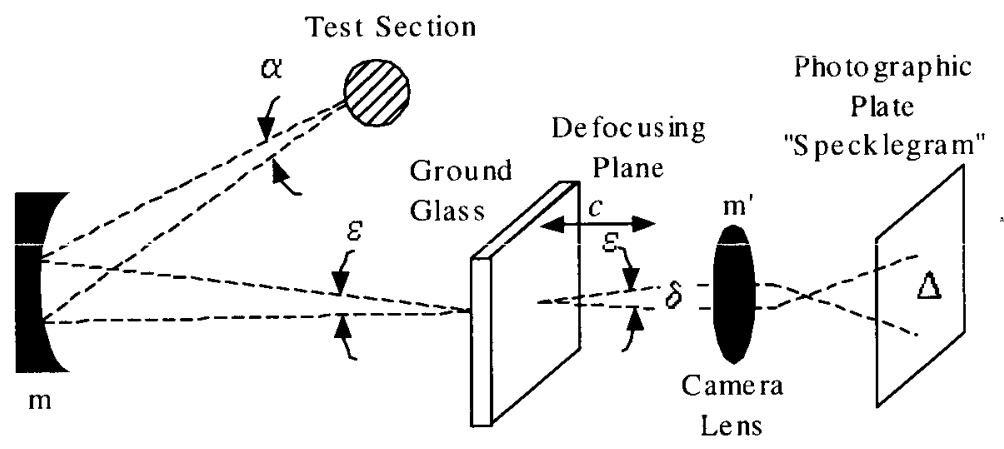

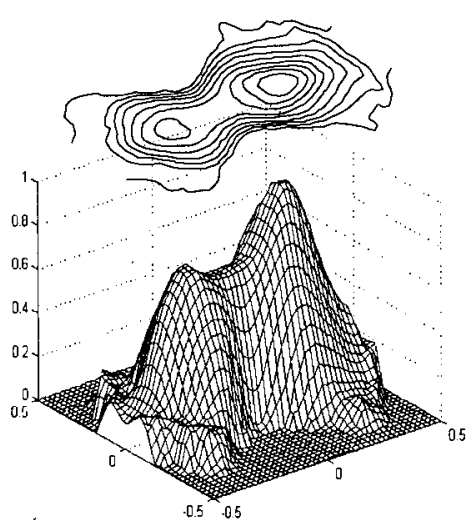

(a)

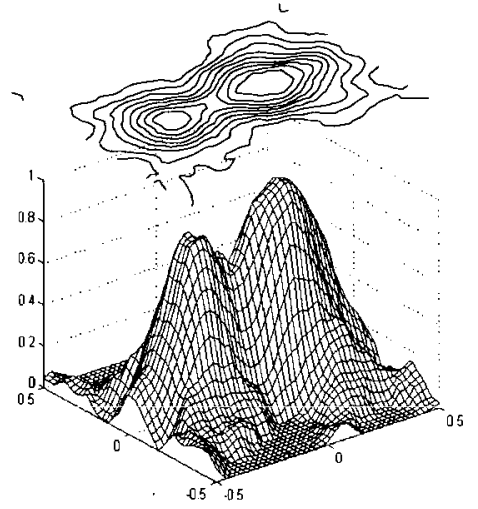

(b)

Figure 4 Reconstructed fields for twohole orifice using (a) modified ART and (b) FC

Figure 3 Geometric formulation of speckle dislocation

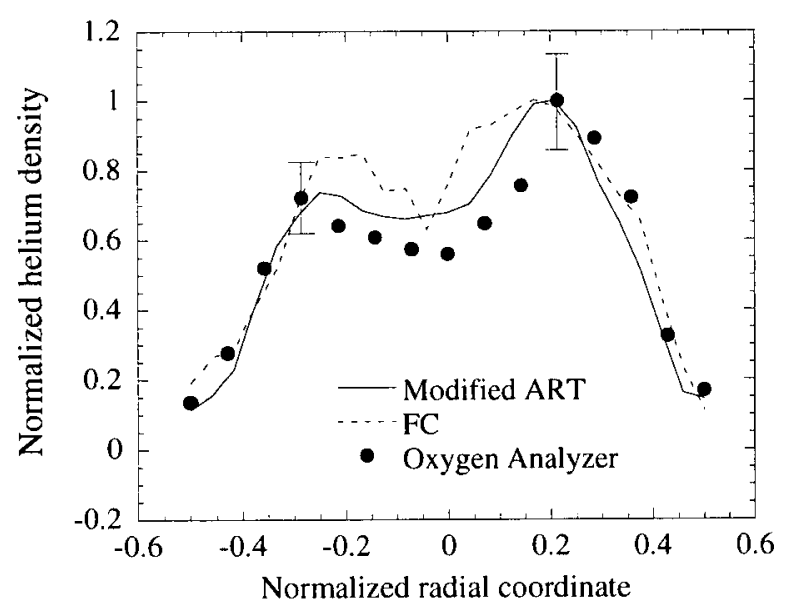

Figure 5 Comparison of normalized helium density distribution along the centerline of two-hole orifice for modified ART, FC, and oxygen analyzer data 